The Astrophysical Journal, 667:000-000, 2007 October 1

(C) 2007. The American Astronomical Society. All rights reserved. Printed in U.S.A.

\title{
TOWARD UNDERSTANDING THE B[e] PHENOMENON. I. DEFINITION OF THE GALACTIC FS CMa STARS
}

\author{
A. S. Miroshnichenko \\ Department of Physics and Astronomy, University of North Carolina, Greensboro, NC 27402; a_mirosh@uncg.edu \\ Received 2007 March 13; accepted 2007 June 11
}

\begin{abstract}
The $\mathrm{B}[\mathrm{e}]$ phenomenon is defined as the simultaneous presence of low-excitation forbidden line emission and strong infrared excess in the spectra of early-type stars. It was discovered in our Galaxy 30 years ago in the course of the early exploration of the infrared sky and initially identified in 65 Galactic objects, of which nearly half remained unclassified. The phenomenon is associated with objects at different evolutionary stages, ranging from the pre-mainsequence to the planetary nebula stage. We review the studies of both the original 65 and subsequently identified Galactic stars with the $\mathrm{B}[\mathrm{e}]$ phenomenon. A new classification is proposed for stars with the $\mathrm{B}[\mathrm{e}]$ phenomenon based on the time of dust formation in their environments. Properties of the unclassified Galactic B[e] stars are analyzed. We propose that these objects are binary systems that are currently undergoing or have recently undergone a phase of rapid mass exchange, associated with a strong mass loss and dust formation. A new name, FS CMa stars, and classification criteria are proposed for the unclassified $\mathrm{B}[\mathrm{e}]$ stars.
\end{abstract}

Subject headings: circumstellar matter — stars: early-type — stars: emission-line, Be

\section{INTRODUCTION}

Among the first discoveries of infrared (IR) astronomy, introduced in the 1960s, was a large class of object, initially called "infrared stars." These stars exhibit a definite excess of IR radiation above the expected photospheric level. Most of them are associated with nebulosities and were initially, although not firmly, suggested to be protostars (Low et al. 1970). One of their first noticed properties was a correlation between the low-excitation Fe II line emission and IR excess (Geisel 1970). It was interpreted as a consequence of a large-scale mass loss that leads to rapid dust formation in the circumstellar (CS) envelopes.

Soon it was realized that $\sim 30 \%$ of the 70 objects reported by Geisel (1970) are indeed young stars, surrounded by dust from their protostellar clouds. Another $\sim 40 \%$ turned out to be evolved objects, such as supergiants (e.g., P Cyg, $\mu$ Cep), planetary nebulae (PNe; e.g., NGC 7027, NGC 6572), and symbiotic stars (e.g., R Aqr) that indeed formed CS dust at different evolutionary stages (e.g., the asymptotic giant branch [AGB] stage or the luminous blue variable $[\mathrm{LBV}]$ stage). The remaining $30 \%$ comprised classical Be stars with gaseous-only envelopes and six other objects (MWC 17, FS CMa, RY Sct, MWC 342, MWC 349, and MWC 645), which a few years later were included in the group of peculiar Be stars, better known as B[e] stars (Allen \& Swings 1976).

Further expansion of IR astronomy toward longer wavelengths along with the development of the theoretical foundation of star formation and evolution have led to the finding of many more stars with CS dusty envelopes, as well as to a clear understanding of their evolutionary state and the CS dust nature. It was established that both early- and late-type emission-line stars with large IR excesses located in star-forming regions (Herbig Ae/Be $[\mathrm{HAeBe}]$ and T Tauri stars, respectively) are pre-main-sequence objects of different initial masses. Later the IRAS mission discovered young main-sequence Vega-type stars with dusty debris disks (Aumann 1985) and proto-planetary nebulae (PPNe) at an intermediate evolutionary stage between the AGB and the PN stage (e.g., van der Veen et al. 1989). Both of these types of objects were found to inherit CS dust from their precursors (HAeBe and AGB stars, respectively). Even earlier, it was shown that CS dust can be formed in the chromospheres and winds of cool stars (e.g., Salpeter 1974). Also, studies of hot luminous dusty objects (WR stars and LBVs) resulted in understanding that CS dust can be formed even in much harsher (e.g., more dust-destroying UV radiation), but special (e.g., high matter density due to strong mass loss and/or clumping), conditions (e.g., van der Hucht et al. 1984).

The described progress in understanding of the CS dust formation and evolution around various objects became possible due to long-term careful studies of individual groups of objects both observationally and theoretically. On this background, it is clearly visible that our understanding of one group, the $\mathrm{B}[\mathrm{e}]$ stars, progressed much slower. In this paper we summarize major points of its investigation, discuss its place in the current picture of stellar evolution, and suggest a new approach to its further studies.

\section{B[e] STARS IN THE 20th CENTURY}

$\mathrm{B}[\mathrm{e}]$ stars were selected as emission-line B-type stars with large IR excesses due to hot CS dust and optical spectra that contain forbidden emission lines (e.g., [O I], [ $\mathrm{Fe}$ II], [N II], and sometimes $[\mathrm{O} \mathrm{III}]$ ) in addition to permitted ones, such as the Balmer and $\mathrm{Fe}$ II lines (Allen \& Swings 1976). The discoverers found that the group-defining features are characteristic of a broad range of objects from Be stars to PNe. They suggested three mechanisms to explain the CS shell formation: "formation of PNe as a single event, interaction of an $\mathrm{OB}$ star with a late-type companion and direct ejection from a particularly massive Be or Oe star."

Follow-up studies confirmed that all the proposed mechanisms work: PNe, symbiotic binaries, and supergiants (including an LBV, $\eta$ Carinae) have been recognized among the group objects. Pre-main-sequence $\mathrm{HAeBe}$ were not supposed to be part of the group, but a few of them were later identified in the original B[e] star list (Finkenzeller \& Mundt 1984). Also nearly half of the group has been associated neither with known types of stellar objects nor with known evolutionary stages due to the following reasons. $\mathrm{B}[\mathrm{e}]$ stars are often significantly reddened in the optical range $[E(B-V) \geq 1 \mathrm{mag}]$, and the reddening removal is uncertain because of the unknown relative contribution of the CS and interstellar (IS) extinction. Photospheric lines have not been detected in the spectra of many $\mathrm{B}[\mathrm{e}]$ stars even at high resolution. 


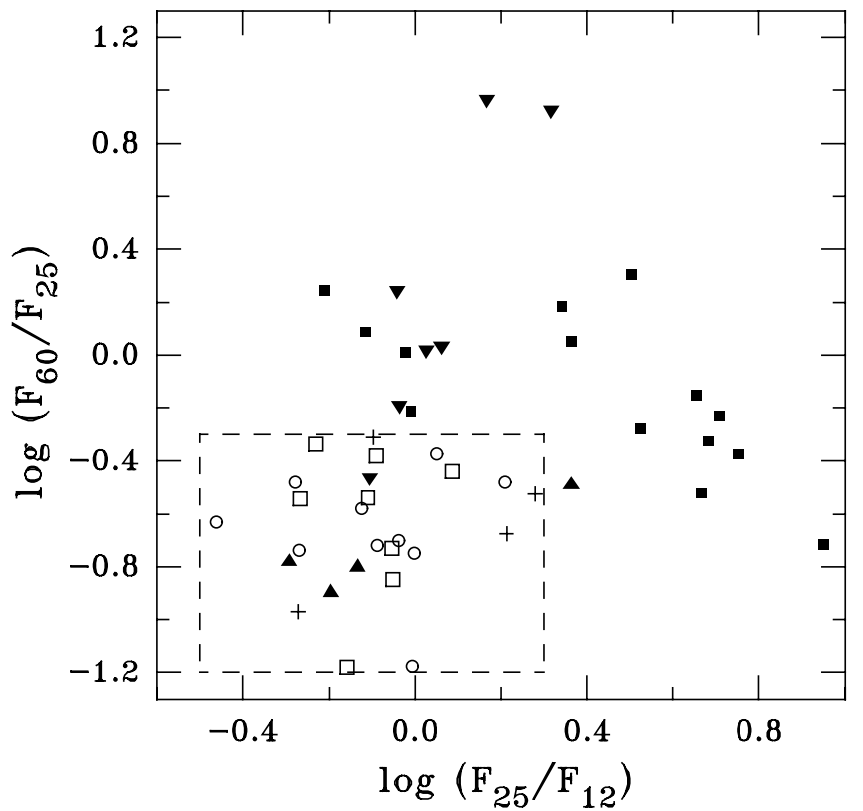

FIG. 1.-IRAS color-color diagram for B[e] stars. Open circles: well-established FS CMa stars and sgB[e] from Allen \& Swings (1976); open squares: well-established FS CMa stars and $\mathrm{sgB}[\mathrm{e}]$ from Dong \& Hu (1991); plus signs: candidate FS CMa stars with typical $60 \mu \mathrm{m}$ fluxes from Table 2; filled upward-pointing triangles: symbB[e]; filled downward-pointing triangles: $\mathrm{HAeB}[\mathrm{e}]$; filled squares: cPNB[e]. The dashed box shows the most probable location of FS CMa stars.

Therefore, their luminosities and spectral types are highly uncertain. As a result, the location in the Hertzsprung-Russel diagram (HRD) and the evolutionary state cannot be well constrained. $\mathrm{B}[\mathrm{e}]$ stars were mostly studied as individual objects and not as a group, probably due to the initially suggested heterogeneity. Even the discovery of B[e] supergiants in the Magellanic Clouds (Zickgraf et al. 1985, 1986) has not triggered more interest in $\mathrm{B}[\mathrm{e}]$ stars in the community.

\subsection{B[e] Stars in the IRAS and MSX Surveys}

The IRAS survey detected Galactic $\mathrm{B}[\mathrm{e}]$ stars from the original list of Allen \& Swings (1976) in one or more photometric bands (44 out of the 65 objects were detected even at $60 \mu \mathrm{m}$ ). Fluxes from only three objects (GG Car, Hen 3-230, and Hen 3-485) were not accurately measured due to their location in crowded regions. Nevertheless, in contrast to the IRAS results for other stellar groups with CS envelopes, these data have not been analyzed for the entire group of $\mathrm{B}[\mathrm{e}]$ stars until recently.

An IRAS color-color diagram for $\mathrm{B}[\mathrm{e}]$ stars is shown in Figure 1. Its striking feature, first noticed by Sheikina et al. (2000), is that a number of $\mathrm{B}[\mathrm{e}]$ stars (within the dashed box) with no evidence for dominating cool companions are characterized by a steep flux decrease toward longer wavelengths. Such an IR spectral energy distribution (SED) is unusual for hot stars that emit enough UV photons to warm even distant CS dust and indicates that the dusty envelopes are compact (see discussion in $\S 3.2$ ).

Unfortunately, due to a wide aperture of the IRAS detectors at $60 \mu \mathrm{m}$ and the presence of contaminating radiation from IS dust (IR cirrus) that is especially strong near the Galactic plane, where the majority of $\mathrm{B}[\mathrm{e}]$ stars are located, the $60 \mu \mathrm{m} I R A S$ fluxes cannot be considered reliable in some cases. Nevertheless, there are enough $\mathrm{B}[\mathrm{e}]$ stars with point-source-like $60 \mu \mathrm{m}$ images or with definitely weak $60 \mu \mathrm{m}$ fluxes to recognize the importance of the group in the dashed box.

In 1996 the $M S X$ survey of the Galactic plane $\left(|b| \leq 5^{\circ}\right)$ in six IR bands at wavelengths between 4 and $21 \mu \mathrm{m}$ and with a sen- sitivity similar to that of IRAS detected $52 \mathrm{~B}[\mathrm{e}]$ stars (Egan et al. 2003). The $M S X$ fluxes turned out to be very similar to those of $I R A S$ at close wavelengths, thus indicating no significant changes in the dusty component of the $\mathrm{B}[\mathrm{e}]$ star envelopes over the 13 years between the surveys. $\mathrm{B}[\mathrm{e}]$ stars are not well separated on MSX color-color diagrams because of a narrow wavelength range covered.

\subsection{Other Sources of Galactic B[e] Stars}

In their search for dusty B-type stars, Allen \& Swings (1976) have surveyed only the brightest objects and/or those with the strongest line emission. The near-IR brightness threshold ( $K \sim 8 \mathrm{mag}$ ) was good at the time, but not deep enough for a more complete search. Nevertheless, there were no direct attempts to expand the list of Galactic B[e] stars, and only two papers turned out to contain new candidates to the group.

Carlson \& Henize (1979) presented a list of 20 southern stars with strong line emission in the optical region. It partially overlapped with the original list of $\mathrm{B}[\mathrm{e}]$ stars (two common objects, CPD -52 9243 and GG Car). Another object (HD 327083) was later shown to be a binary system with a $B[\mathrm{e}]$ supergiant (Miroshnichenko et al. 2003b). Another important list was published by Dong \& Hu (1991), who positionally cross-correlated the catalog of Galactic early-type emission-line stars (Wackerling 1970) and the IRAS Point Source Catalog (PSC; IPAC 1986). These authors found nearly 200 objects with strong IR excesses ( $V-[25] \geq 8 \mathrm{mag}$, where [25] is the magnitude in the $25 \mu \mathrm{m}$ IRAS band). The list included 12 original $\mathrm{B}[\mathrm{e}]$ stars ( $\eta$ Car, $\mathrm{RY}$ Sct, CI Cam, FS CMa, HD 50138, HD 87643, MWC 342, MWC 623, MWC 645, MWC 922, AS 222, and AS 225) and five objects that were later identified as B[e] stars (AS 78, AS 381, Hen 3-298, Hen 3-303, and MWC 657; Miroshnichenko et al. 2002a). Also, Dong \& Hu (1991) have identified all three B[e] objects from Carlson \& Henize (1979) mentioned above. On the other hand, $\sim 30 \%$ of the IR sources were identified incorrectly by Dong \& Hu (1991) due to a low positional accuracy of the IRAS $\operatorname{PSC}\left(10^{\prime \prime}-30^{\prime \prime}\right)$. It can easily be shown using recently released allsky data catalogs (e.g., USNO-B1.0 and 2MASS) with a much higher astrometric accuracy $\left(1^{\prime \prime}-2^{\prime \prime}\right)$. In any case, these studies showed that the $\mathrm{B}[\mathrm{e}]$ group is larger than the original list by Allen \& Swings (1976).

\subsection{First Classification of B[e] Stars}

Summarizing the first 20 years of studies of B[e] stars, Lamers et al. (1998, hereafter L98) suggested an evolutionary classification by recognizing five subgroups: pre-main-sequence $\mathrm{HAeB}[\mathrm{e}]$ stars, symbiotic binaries (a cool giant and a white dwarf or a neutron star, symbB[e]), compact PPNe/PNe (cPNB[e]), supergiant ( $\mathrm{sgB}[\mathrm{e}]$ ), and unclassified (unclB[e]). L98 concluded that the $\mathrm{B}[\mathrm{e}]$ phenomenon is associated with stars at very different evolutionary stages, but with similar conditions in their gaseous and dusty envelopes. These conditions include (1) a large emission measure of singly ionized gas $\left(\mathrm{EM} \geq 10^{56} \mathrm{~cm}^{-3}\right.$ ) to account for the presence of strong Balmer lines; (2) CS gas temperatures of $\sim 10^{4} \mathrm{~K}$ to account for the presence of low-excitation permitted emission lines, such as Fe II; and (3) large geometrical extension of the CS material that provides a sufficient amount of gas to account for the presence of forbidden emission lines and a sufficient density in its outermost regions to account for the presence of CS dust. L98 also pointed out that the CS envelope geometry is most probably disklike, which naturally provides conditions for a high density of the CS material and, as a consequence, for shielding of CS dust from the ionizing stellar radiation. This paper also contains identification criteria for each of the $\mathrm{B}[\mathrm{e}]$ subgroups, except 
TABLE 1

FS CMa Stars and Galactic B[e] Supergiants with Known Fundamental Parameters

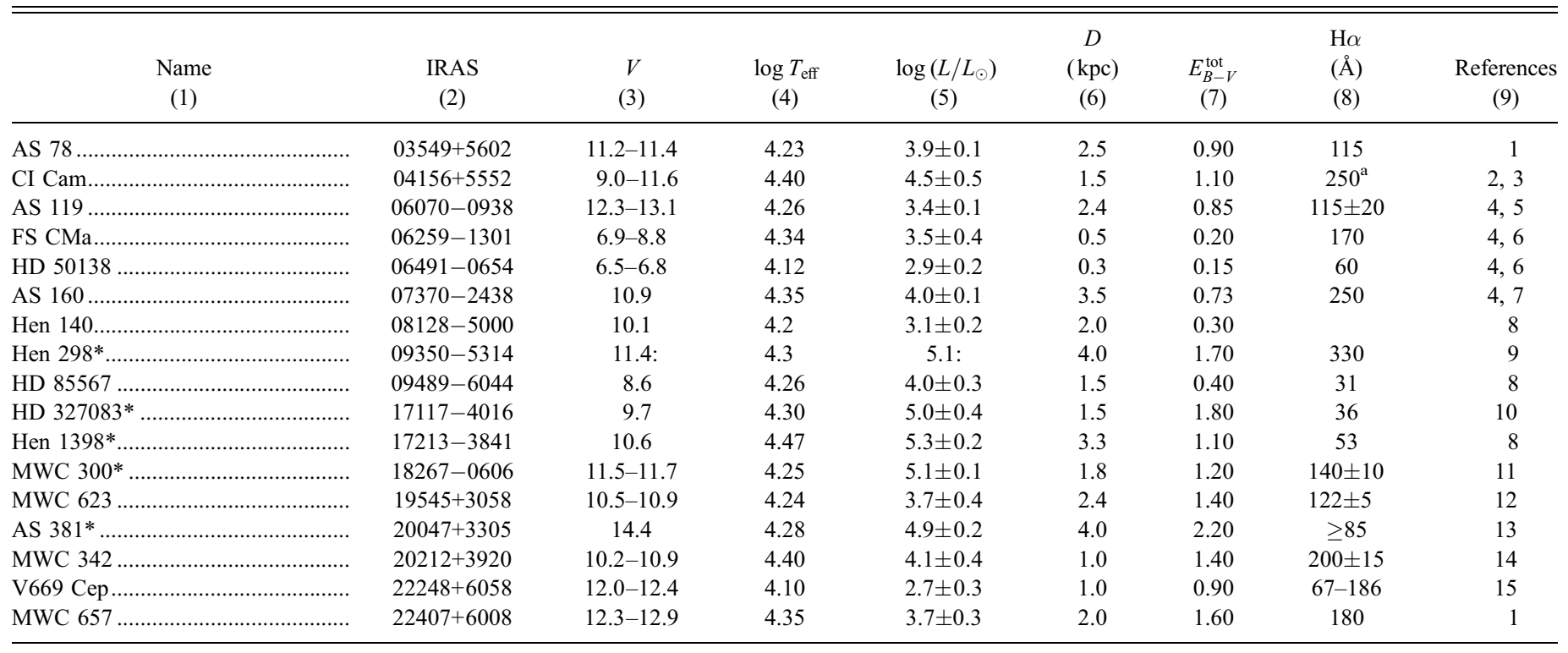

Notes.—Col. (1): Object names; $\mathrm{sgB}[\mathrm{e}]$ are shown with an asterisk. Col. (2): Their IRAS IDs. Col. (3): Detected range of visual brightness. Cols. (4) and (5): Surface temperature and luminosity of the star or the hot companion (in case of known binaries). Col. (6): Distance toward the star/binary. Col. (7): Total (IS plus CS) reddening. Col. (8): Equivalent width of the $\mathrm{H} \alpha$ line. Col. (9): References to the data.

a A typical line strength in the quiescent state; see Clark et al. (2000).

References.- (1) Miroshnichenko et al. 2000; (2) Miroshnichenko et al. 2002b; (3) Robinson et al. 2002; (4) Cidale et al. 2001; (5) Miroshnichenko et al. 2006; (6) L98; (7) Miroshnichenko et al. 2003a; (8) Miroshnichenko et al. 2001; (9) Miroshnichenko et al. 2005; (10) Miroshnichenko et al. 2003b; (11) Miroshnichenko et al. 2004; (12) Zickgraf 2001; (13) Miroshnichenko et al. 2002c; (14) Miroshnichenko \& Corporon 1999; (15) Miroshnichenko et al. $2002 \mathrm{~d}$.

for the unclB[e] stars, whose nature and evolutionary state have not been revealed.

L98 became the primary reference for studies of objects with the $\mathrm{B}[\mathrm{e}]$ phenomenon. Nevertheless, their analysis has not led to identification of new such objects. Also, L98 have not considered the possible nature of unclB $[\mathrm{e}]$ in detail. They only discussed two objects with nebulae (MWC 349 and HD 87643) and two objects without nebulae (FS CMa and HD 50138), trying to summarize the existing data and classify them as either $\mathrm{HAeB}[\mathrm{e}]$, extreme classical Be, or massive evolved objects. Moreover, neither L98 nor other studies, published before 2000, have analyzed the IR $\mathrm{SEDs}$ of $\mathrm{B}[\mathrm{e}]$ stars and considered reasons for dust formation as a classification criterion.

\section{CLASSIFICATION OF B[e] STARS BASED ON THE DUST FORMATION TIME}

Let us consider the nature of CS dust in the subgroups of $\mathrm{B}[\mathrm{e}]$ stars suggested by L98. CS dust is derived from the previous generations of stars in $\mathrm{HAeB}[\mathrm{e}]$ (i.e., has protostellar nature). In $\mathrm{cPNB}[\mathrm{e}]$, it is left from the previous AGB evolutionary stage. Therefore, objects of these two subgroups are not directly responsible for the dust formation.

In symbB[e], CS dust is formed in the ejecta of cool giants, as in AGB stars. In $\mathrm{sgB}[\mathrm{e}]$, which are very luminous objects with extremely dense radiatively driven winds, dust can be formed due to the presence of heavy elements produced in their interiors and selfshielding of parts of their winds (e.g., due to clumping) from dustdestroying UV radiation. This is similar to the situation in WR stars and LBVs. Finally, as shown in $\S 4$, it is very likely that the CS dust formation either is ongoing or has recently ended in unclB $[\mathrm{e}]$.

Therefore, two large classes of stars with the $\mathrm{B}[\mathrm{e}]$ phenomenon can be recognized depending on the CS dust formation time: ongoing/recent formation and formation during previous stage(s) of evolution. Among the dust-forming objects, symbB[e] seem to be different from $\mathrm{sgB}[\mathrm{e}]$ and unclB[e]. In $\operatorname{symbB}[\mathrm{e}]$, we deal with strong mass loss from Roche lobe filling cool companions in close binary systems (see Zamanov et al. 2006), conditions in whose CS envelopes may be very similar to those in dust-forming AGB stars. In $\mathrm{sgB}[\mathrm{e}]$ and unclB[e], the B-type star/companion seems to be responsible for mass loss, and it is not obvious whether known binary systems of these two subgroups are contact (with the exception of RY Sct). Therefore, we argue that $\mathrm{sgB}[\mathrm{e}]$ and unclB[e] represent a distinct group of dust-forming hot stars.

\subsection{High-Luminosity Dust-forming B[e] Stars}

Although a few sgB[e] were introduced by Allen \& Swings (1976), they were shown to be a large group in the Magellanic Clouds by Zickgraf et al. (1986). L98 listed all sgB[e] in the LMC/SMC (15 objects; for a recent review see also Zickgraf 2006) and suggested two most probable Galactic sgB[e] candidates (CPD -52 9243 and GG Car) and three unclear ones (HD 87643, MWC 300, and MWC 349).

Two other objects from the original list of B[e] stars, RY Sct (see Men'shchikov \& Miroshnichenko 2005) and 3 Pup (Stee et al. 2004), have also been shown to be supergiants. A few more objects have recently been added to the Galactic population of sgB[e]: Hen 3-1398 (Miroshnichenko et al. 2001), AS 381 (Miroshnichenko et al. 2002c), HD 327083 (Miroshnichenko et al. 2003b), and Hen 3-298 (Miroshnichenko et al. 2005). The luminosity of MWC 300 was better constrained (see Table 1), and so was its $\operatorname{sgB}[\mathrm{e}]$ status. On the other hand, the status of MWC 349 (for different opinions see Hofmann et al. 2002; Meyer et al. 2002) is still uncertain. Also, luminosities of CI Cam, GG Car, CPD - 52 9243, CPD - 57 2874, and MWC 137, which have been considered to be high-luminosity objects, need further refinement. 


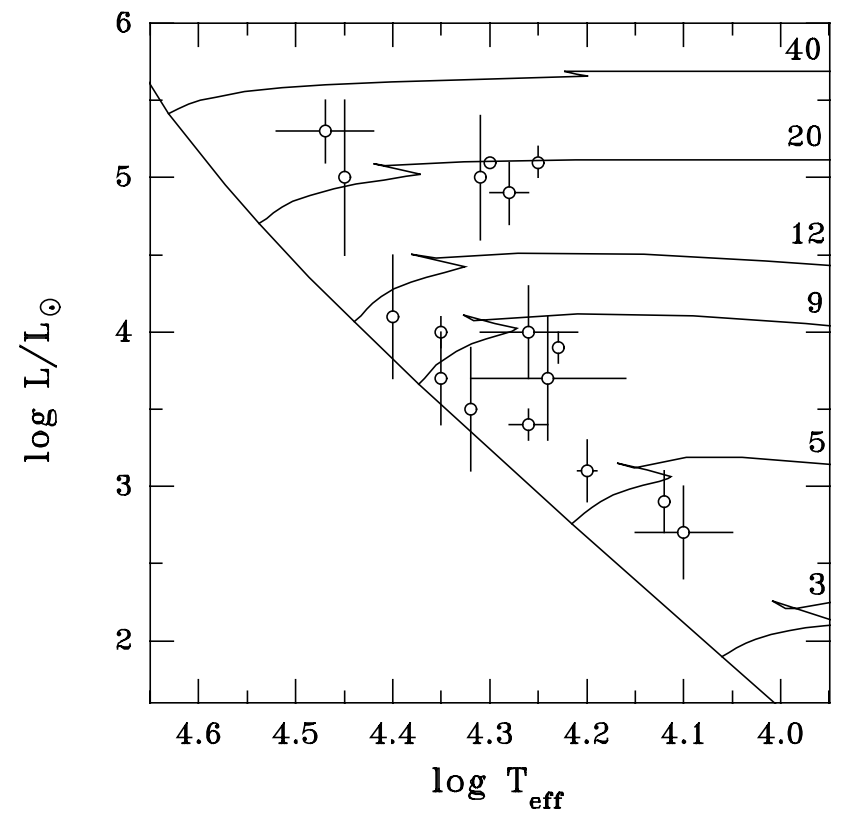

FIG. 2.- HRD for FS CMa-type stars and Galactic B[e] supergiants. The solid lines show the zero-age main-sequence and evolutionary tracks for single stars (Schaller et al. 1992) with the initial masses indicated in $M_{\odot}$. Fundamental parameters are listed in Table 1 . The five Galactic $\operatorname{sgB}[\mathrm{e}]$ with well-constrained fundamental parameters are located above $\log \left(L / L_{\odot}\right) \sim 4.7$.

There are two interesting differences between the $\operatorname{sgB}[\mathrm{e}]$ of the Milky Way and those of the Magellanic Clouds. Summarizing studies of the Galactic sgB[e], Miroshnichenko (2006) showed that their maximum luminosity is $\log \left(L / L_{\odot}\right)=5.1 \pm 0.2$, while half of the LMC/SMC $\operatorname{sgB}[\mathrm{e}]$ are more luminous. Also, most of the members and candidates to the Galactic $\mathrm{sgB}[\mathrm{e}]$ are either recognized (RY Sct, CI Cam, HD 327083, and AS 381) or suspected (MWC 300, 3 Pup, MWC 349, MWC 137, and GG Car) binaries, while only two binaries have been found among the $\mathrm{LMC} / \mathrm{SMC}$ sgB[e] (RMC 4 and HD 34664; Zickgraf 2006). Both of these features may partially be selection effects, but they certainly deserve further study.

The mechanisms of the very strong mass loss from $\mathrm{sgB}[\mathrm{e}]$ are still unclear (see reviews by Meynet \& Maeder 2006; Hillier 2006). However, the locations in the HRD (see Fig. 2 for the Galactic $\operatorname{sgB}[\mathrm{e}]$ ) indicate that $\mathrm{sgB}[\mathrm{e}]$ are evolved objects that are naturally capable of efficiently losing mass. An interesting hypothesis about formation of $\mathrm{sgB}[\mathrm{e}]$ by mergers in close binaries was recently suggested by Podsiadlowski et al. (2006). In any case, $\mathrm{sgB}[\mathrm{e}]$ seem to expel so much material that dust formation is definitely possible in their CS envelopes (e.g., Bjorkman 1998; Kraus \& Lamers 2003).

\subsection{Nonsupergiant Dust-forming B[e] Stars (FS CMa Stars)}

UnclB [e] objects, especially the brightest ones (e.g., FS CMa and HD 50138), have only been studied in detail individually with yet controversial conclusions about their nature and evolutionary state. For example, Sitko et al. (1994) argue that FS CMa is a pre-main-sequence star that experienced evaporation of a protocomet, responsible for the star's $15 \mathrm{yr}$ long fading. Herbig (1994) declines the young age due to its isolation from starforming regions. Cidale et al. (2001) try to prove that FS CMa is a single star based on its fundamental parameters, measured Balmer jump, and the absence of companion features in the spectrum. At the same time, Baines et al. (2006) detected a faint secondary com- panion in this system using spectroastrometry. A similar discussion applies to a number of other unclB[e] (e.g., HD 50138, MWC 17, and MWC 342).

Some progress in studies of unclB[e] was made when Sheikina et al. (2000) analyzed IRAS colors of $\mathrm{B}[\mathrm{e}]$ stars. This analysis of the original $\mathrm{B}[\mathrm{e}]$ group, as well as of the objects found by Carlson \& Henize (1979) and Dong \& Hu (1991), prompted Miroshnichenko et al. (2002a) to suggest a new distinct subgroup of $\sim 20 \mathrm{~B}[\mathrm{e}]$ stars with a sharp decrease of the IR flux longward of $\sim 10-30 \mu \mathrm{m}$ $\left[-0.5 \leq \log \left(F_{25} / F_{12}\right) \leq 0.3,-1.1 \leq \log \left(F_{60} / F_{25}\right) \leq-0.3\right.$, where $F_{12}, F_{25}$, and $F_{60}$ are the fluxes $\left(F_{\nu}\right)$ in the IRAS photometric bands centered at 12,25 , and $60 \mu \mathrm{m}$, respectively; see Fig. 1].

Another distinct feature of this subgroup is extremely strong emission-line spectra. On average, Balmer lines of its members are an order of magnitude stronger than those in Be stars and even in hot supergiants and hypergiants (see Table 1). Initially, this subgroup included both $\mathrm{sgB}[\mathrm{e}]$ and $\mathrm{unclB}[\mathrm{e}]$ and was tentatively called $\mathrm{B}[\mathrm{e}]$ stars with warm dust $(\mathrm{B}[\mathrm{e}] \mathrm{WD})$. This name can be found in the literature since 2002 and is obviously confusing because WD has stood for white dwarfs since long ago. Time has certainly come to put an end to this confusion.

FS CMa stars are located in a region of the IRAS color-color diagram mostly occupied by cool dusty stars (such as symbiotic and VV Cep-type binaries, as well as M-type stars at the AGB). The latter barely emit UV radiation, which is most efficiently absorbed by dust. Therefore, they are capable of heating only the closest dust, even if their envelopes are extended. Hot stars may heat more distant dust and, as a result, have flatter IR SEDs. Therefore, spatial distribution of CS dust near FS CMa stars should sharply fall off with distance (as $\sim r^{-2}$ or faster). SED modeling of some FS CMa stars (Ivezić et al. 1998) showed that their dusty envelopes are much more compact than those of young stars, even if the dust is spherically distributed. If the dust distribution is disklike, which is most likely the case for these objects (e.g., L98), the envelopes should be even more compact due to a higher optical depth and lower temperatures of the dust compared to the spherical case (Miroshnichenko et al. 1999). A most likely explanation of such a CS dust distribution is recent formation of the envelopes.

Since the discovery of B[e] stars, FS CMa has been called a prototype of this kind of object (Allen \& Swings 1976; Swings 2006). Its location in the HRD indicates that it is not a supergiant. Therefore, in order to separate $\mathrm{sgB}[\mathrm{e}]$ from less luminous objects within the main sequence, which might have a different nature of the $\mathrm{B}[\mathrm{e}]$ phenomenon, and replace the old confusing names (unclB[e] and $\mathrm{B}[\mathrm{e}] \mathrm{WD}$ ), we propose to call nonsupergiant objects of the above-described new subgroup FS CMa stars.

The above-presented analysis suggests a set of classification criteria for FS CMa stars, all of which must be satisfied simultaneously, as follows:

\section{Observational criteria:}

a) Emission-line spectra contain the following: hydrogen lines that are typically stronger than those in the spectra of $\mathrm{Be}$ stars, $\mathrm{HAeBe}$, and normal supergiants; $\mathrm{Fe}$ II and [ $\left.\mathrm{O}_{\mathrm{I}}\right]$ lines; and sometimes [Fe II] lines and weak [O III] lines.

b) A large IR excess that peaks at 10-30 $\mu \mathrm{m}$ and sharply decreases longward (location in the dashed box in the IRAS color-color diagram for objects with all three reliably measured IRAS fluxes).

c) Location outside of star-forming regions.

d) When present, a secondary companion (i) is typically fainter and cooler than the primary (if a normal star) and (ii) is degenerate. 
TABLE 2

FS CMa-Type Star Candidates

\begin{tabular}{|c|c|c|c|c|c|}
\hline $\begin{array}{l}\text { Name } \\
\text { (1) }\end{array}$ & $\begin{array}{l}\text { IRAS } \\
\text { (2) }\end{array}$ & $\begin{array}{l}V \\
(3)\end{array}$ & $\begin{array}{l}\mathrm{H} \alpha \\
(\AA) \\
(4)\end{array}$ & $\begin{array}{l}\text { References } \\
\text { (5) }\end{array}$ & $\begin{array}{c}I R A S 60 \mu \mathrm{m} \\
\text { (6) }\end{array}$ \\
\hline MWC $17 \ldots \ldots \ldots \ldots . . . . . .$. & $01441+6026$ & 13.2 & 680 & 1 & Typical \\
\hline MWC $819 \ldots \ldots \ldots . . . . .$. & $06420+0122$ & 15.3 & $\ldots$ & 2 & Extended \\
\hline He 2-91 .................. & $13068-6255$ & 14.4 & $\ldots$ & 3 & Contaminated \\
\hline Pe $2-9 \ldots \ldots \ldots \ldots$ & $16212-4836$ & 17 & $\ldots$ & 4 & Weak \\
\hline Hen 3-1227 ............ & $16348-4517$ & 13.3 & $\ldots$ & 3 & Contaminated \\
\hline AS $222 \ldots \ldots \ldots$ & $17108-3855$ & 13 & $\ldots$ & 3 & Extended \\
\hline AS $225 \ldots \ldots \ldots \ldots$ & $17175-3757$ & 12.5 & $\ldots$ & 3 & Contaminated \\
\hline MWC $922 \ldots \ldots \ldots \ldots . . . .$. & $18184-1302$ & 14.3 & $\ldots$ & 3 & Typical \\
\hline MWC $939 \ldots \ldots \ldots \ldots . . .$. & $18313-1738$ & 12.4 & 390 & 1 & Typical \\
\hline SS $170 \ldots \ldots \ldots \ldots \ldots$ & $18316-0028$ & 14 & $\ldots$ & 3 & Weak \\
\hline М 1-76 .... & $20145+3656$ & 14.7 & $\ldots$ & 5 & Typical \\
\hline MWC $645 \ldots \ldots \ldots . . . . .$. & $21516+5245$ & 12.5 & 218 & 1 & Typical \\
\hline MWC $1055 \ldots \ldots \ldots . . .$. & $22065+5358$ & 12.3 & 181 & 1 & Typical \\
\hline Hen $3-230 \ldots \ldots \ldots . . . .$. & $\ldots$ & 12.3 & 229 & 1,3 & Contaminated \\
\hline Hen $3-485 \ldots \ldots \ldots \ldots . . .$. & $\ldots$ & 11.6 & 165 & 1,3 & Contaminated \\
\hline
\end{tabular}

Notes.-Col. (1): Object names. Col. (2): Their IRAS IDs. Col. (3): Visual brightness. Col. (4): Equivalent width of the $\mathrm{H} \alpha$ line. Col. (5): References to the data. Col. (6): Qualitative description of the IRAS $60 \mu \mathrm{m}$ data. The description in col. (6) means the following: typical - the $60 \mu \mathrm{m}$-to- $25 \mu \mathrm{m}$ flux ratio is typical of FS CMa stars; weak - the flux is too weak to be reliably measured and is much lower than the $25 \mu \mathrm{m}$ flux; contaminated - the IRAS source is observed on a complicated background that does not allow reliable flux measurement; extended - the IRAS source does not fit the point-source template and the flux can be overestimated.

References.-(1) Zickgraf 2003; (2) Landaberry et al. 2001; (3) Pereira et al. 2003; (4) Stenholm \& Acker 1987; (5) Sabbadin \& Bianchini 1979.

\section{Physical criteria:}

a) $T_{\text {eff }}$ of the hot star/companion between $\sim 9000$ and $\sim 30,000 \mathrm{~K}$ (O9-A2 type stars).

b) Luminosity of the hot star/companion $\log \left(L / L_{\odot}\right)$ between $\sim 2.5$ and $\sim 4.5$.

These criteria separate FS CMa stars almost uniquely. The only confusion may occur with intermediate-mass post-AGB stars (see $\S 4.1$ ) and with $\operatorname{sgB}[\mathrm{e}]$ due to uncertain luminosity. Note that the only hot star other than FS CMa or $\mathrm{sgB}[\mathrm{e}]$ in the dashed box in Figure 1 is HD 190073, an unusual isolated HAeBe candidate (Pogodin et al. 2005). Nevertheless, only careful studies of individual objects can reveal their true nature. Further investigation of the group may reveal more constraints on both the observed and physical properties of its objects.

How large is the group of FS CMa stars? As was shown above, the main problems with classifying these objects are an uncertain $60 \mu \mathrm{m}$ flux and an uncertain luminosity. Therefore, here we list separately the well-established members, which have been shown to satisfy all the selection criteria, and candidates, which only partially meet the selection criteria and are not well-established members of any other class of object. The former part includes 11 objects from Miroshnichenko et al. (2002a): AS 78, FS CMa, HD 50138, AS 160, Hen 3-140, Hen 3-303, HD 85567, MWC 623, MWC 342, V669 Cep, and MWC 657. The latter part (listed in Table 2) contains objects from the original list (Allen \& Swings 1976) with the IRAS colors from the dashed box in Figure 1 or those rejected as $\mathrm{PNe}$ or $\mathrm{HAeBe}$. In addition, a few $\mathrm{sgB}[\mathrm{e}]$ candidates with uncertain luminosities can be considered FS CMa star candidates. These include CI Cam, whose luminosity has been recently shown to be $\sim 10^{4} L_{\odot}$ (Miroshnichenko et al. 2002b; Barsukova et al. 2006), as well as CPD -52 9243,
CPD -57 2874, and MWC 137, whose high luminosities have not been confirmed yet.

Thus, the FS CMa star group contains 30 objects (including candidates) from Allen \& Swings (1976) and Dong \& Hu (1991). The group includes almost all former unclB[e] except for a few most poorly studied (e.g., Hen 3-664) or controversial ones (e.g., Ve 2-27). A number of new candidates were introduced by Miroshnichenko et al. (2003c) as a result of a cross-correlation of the IRAS PSC, the 2MASS catalog (Cutri et al. 2003), and the USNO-B1.0 catalog (Monet et al. 2003). A detailed study of these objects will be presented in the next paper of this series.

Below we analyze observational properties of FS CMa stars in more detail in order to put some constraints on their nature and evolutionary state and justify the rejection of the old name "unclassified."

\section{NATURE AND EVOLUTIONARY STATE OF THE FS CMa STARS}

\subsection{Evolutionary State}

In one of the first papers on FS CMa stars, Miroshnichenko et al. (2000) tried to show that they are neither pre-main-sequence nor post-AGB objects. The discussion has not touched all possible aspects of the problem. Here more arguments are considered.

Pre-main-sequence stars exhibit much stronger far-IR excesses (due to cold preexisting dust) and retain them much longer than the near-IR ones (Miroshnichenko et al. 1996; Malfait et al. 1998). The only possibility for a pre-main-sequence star to lose the cold dust first is to be exposed to stellar winds from nearby stars in dense star-forming regions (Hollenbach \& Adams 2004). In contrast, FS CMa stars are isolated objects. L98 considered a possibility that unclB $[\mathrm{e}]$ could be isolated $\mathrm{HAeBe}$, introduced by Grinin et al. (1991). Nevertheless, isolated HAeBe are mostly A-type stars with "flat" IR excesses, typical of pre-mainsequence objects. Moreover, they exhibit irregular strong days to weeks long brightness variations (known as Algol-like minima) that are associated with an optical reddening and increase of the linear polarization. This phenomenon is interpreted as eclipses of the star by density enhancements in the dusty disk (for a discussion see Grinin et al. 1991). Even the most well-observed FS CMa stars (e.g., FS CMa, HD 50138, and MWC 342) do not show such a phenomenon. Their brightness variations can be large (up to $\sim 2 \mathrm{mag}$ ), but they occur on a much longer timescale of at least years, suggesting a different nature than that in isolated HAeBe. Finally, FS CMa stars have significantly stronger emission-line spectra that cannot be explained by typical mass-loss/accretion rates in HAeBe $\left(10^{-8}\right.$ to $10^{-7} M_{\odot} \mathrm{yr}^{-1}$; Grady et al. 1996).

Massive post-AGB stars evolve so fast that their spectral changes due to an increase in $T_{\text {eff }}$ can be detected on a timescale of a decade or so (Blöcker 1995). Their extremely hot photospheres cause strong [O III] emission lines. FS CMa stars are not known to show significant spectral changes, and only the hottest group members show weak [O III] emissions (e.g., MWC 17). Moreover, most post-AGB stars show more pronounced far-IR excesses. The place of FS CMa stars in the IRAS color-color diagram has no overlap with that of post-AGB and PN (Suárez et al. 2006). In addition, post-AGB objects exhibit supergiant-like optical spectra due to their low surface gravity, while photospheric line profiles and HRD locations of FS CMa stars are typical of mainsequence stars.

Low-mass post-AGB stars (known as RV Tau stars) evolve too slow to become hot stars while still retaining hot dust formed during the AGB phase. They may have IRAS colors similar to those of FS CMa stars, but they are easily separated by lower 
surface temperatures. Intermediate-mass post-AGB stars may have optical spectra and IR SEDs similar to those of FS CMa stars (e.g., M2-56; Castro-Carrizo et al. 2002). This case needs further investigation, as the number of well-studied such postAGB objects is still small. However, the latter show easily detectable CO line emission that is not observed in FS CMa stars.

Therefore, it seems very likely that FS CMa stars are still on or close to the main sequence as is indicated by their HRD locations (see Fig. 2).

\subsection{Nature \\ 4.2.1. Single Stars}

Studies of individual FS CMa stars attempted to interpret their unusual properties in the framework of both single star and binary system models. Since secondary companions are not easily detectable in FS CMa stars and even known binaries of this type do not show obvious mass exchange, it seems natural to try to explain their emission-line spectra by mass loss from single hot stars.

Typical mass-loss rates of O-type stars and B-type supergiants strongly depend on the stellar mass and luminosity (de Jager et al. 1988; Vink et al. 2001) and are above $10^{-7} M_{\odot} \mathrm{yr}^{-1}$. Lower luminosity classical Be stars are fast rotators, lose mass at a rate that is at least an order of magnitude lower (Prinja 1989), and have disklike gaseous envelopes. FS CMa stars neither rotate fast nor are luminous, but most of them have double-peaked Balmer line profiles typical of disks.

Mass-loss rates have only been published for two $\mathrm{B}[\mathrm{e}]$ stars with P Cyg line profiles under the assumption of no matter accumulation in a spherical CS envelope. These objects are the $\mathrm{sgB}[\mathrm{e}]$ candidate HD 87643 (de Freitas Pacheco et al. 1982) and AS 78 (Miroshnichenko et al. 2000). In both cases the mass-loss rates were found to be of the order of $10^{-6} M_{\odot} \mathrm{yr}^{-1}$. This is $\sim 2$ orders of magnitude above those expected from radiatively driven winds for stars with the fundamental parameters, which can be estimated from the location of FS CMa stars in the HRD (Vink et al. 2001). In general, emission-line spectra reflect the amount of ionized matter in the CS envelope that could have been accumulated over a long period of time. Therefore, mass-loss rates derived even from P Cyg profiles, which do not necessarily imply a spherical matter distribution, may be significantly overestimated. Nevertheless, it is clear that FS CMa stars are surrounded by dense CS envelopes/disks that contain more matter than those of Be stars and most supergiants. Currently theory of stellar evolution cannot explain such a strong mass loss from a single, not rapidly rotating star.

\subsubsection{Binary Systems}

The above analysis suggests an alternative explanation to the nature of FS CMa stars: most of them, if not all, may be binary systems. First of all, a noticeable fraction of these objects are either already recognized or suspected binaries. Two of them show signatures of a K-type (MWC 623 and V669 Cep) or a degenerate (neutron star or black hole; e.g., CI Cam) companion. Another two exhibit radial velocity or regular brightness variations that may be attributed to orbital motion (AS 160 and MWC 342). A few others show peculiar spectral line profiles and positional differences between emission and absorption features (AS 78, MWC 657, and Hen 3-1398). Finally, FS CMa itself, HD 50138, and HD 85567 have been found to have companions by spectroastrometry (Baines et al. 2006). The latter object was earlier suspected in binarity due to spectroscopic peculiarities (Miroshnichenko et al. 2001).

Also, in binaries, CS gas may accumulate in the companions' Roche lobes and/or in the circumbinary area, thus making the CS matter density sufficient for dust formation and requiring smaller mass-loss rates to explain their strong emission-line spectra. It is worth noting that $\operatorname{sgB}[\mathrm{e}]$, which should naturally have stronger stellar winds than lower luminosity FS CMa stars, do not possess the highest IR excesses and therefore the largest amount of CS dust (see Miroshnichenko 2006). This is another indirect evidence in favor of the matter accumulation in the circumbinary area of FS CMa stars.

In all known binary FS CMa stars except for MWC 623 (Zickgraf 2001), the secondaries are much fainter $(\Delta V \geq 2 \mathrm{mag}$ ) than the B-type primaries. The situation here is similar to that in Be stars, whose binary nature has been proposed at the time of the discovery of the $\mathrm{B}[\mathrm{e}]$ stars (Křiž \& Harmanec 1975). A large brightness ratio of the companions in binary Be stars hampers detection of the secondaries, and only long-term high-resolution spectroscopy is capable of detecting the orbital motion. We monitor a number of FS CMa stars spectroscopically and will report our results in follow-up papers of this series.

Theoretical studies of intermediate-mass binaries (e.g., Wellstein et al. 2001) show that systems with certain companion mass ratios and separations have periods of rapid mass transfer $\left(\sim 10^{-3} M_{\odot} \mathrm{yr}^{-1}\right)$, when the mass gainer cannot accept a large fraction of the incoming matter. The latter can be lost from the system and/or form a circumbinary envelope. FS CMa stars could be good candidates to verify these results. They might also be transitional objects between binary Be stars and symbiotic stars, whose cool primaries are much more luminous than the hot secondaries.

No companion in known binary FS CMa stars seems to fill its Roche lobe; therefore, the systems are currently detached. However, it does not mean that there is no ongoing mass transfer in them. Studies of some detached binary systems with both circular (e.g., $\pi$ Aqr; Bjorkman et al. 2002) and eccentric (e.g., HD 200775; Pogodin et al. 2004) orbits show that companions can exchange mass through their CS disks. Additional mass loss from the system cannot be excluded as well. On the other hand, it seems reasonable to suggest that some FS CMa stars can be some kind of contact $\beta$ Lyr-type binaries.

Studies of known dust-forming B[e] binaries (e.g., Men'shchikov \& Miroshnichenko 2005), as well as simple calculations of the dust temperatures under radiative equilibrium, indicate that CS dust is unlikely to form in the companions' Roche lobes. Since the dust is mostly hot, it still forms or has recently formed around the binary. Otherwise, stellar winds of the companions would move the dust away, therefore making it colder. Thus, the combination of the strong emission-line spectra and strong IR excesses in FS CMa stars (if they are binaries) suggests that they are currently undergoing or have recently finished a rapid mass transfer phase of evolution. Other hypotheses can be considered after a closer study of the entire group.

\section{CONCLUSIONS}

Summarizing our findings, we emphasize that FS CMa stars are not the original "zoo" of various emission-line objects with dusty environments, but most likely are a homogeneous group of possible binaries at a phase of ongoing or recently ended rapid mass transfer. Nearly $30 \%$ of them are either suspected or recognized binary systems. Their main distinct features are extreme amounts of CS gas, which causes the strong emission-line spectra, and a CS dust distribution, which causes the IR flux to steeply fall longward of $10-30 \mu \mathrm{m}$. They are therefore among the most complicated objects because both the gaseous and dusty $\mathrm{CS}$ regions need to be taken into account while interpreting the emergent continuum, spectral line profiles, and SED. In addition, 
binarity can make the CS structure even more complicated compared to those of single stars.

Since most FS CMa stars are relatively distant objects (see Table 1), it would be hard to prove their binarity even with current interferometric capabilities (the expected companion separations are $\sim 1$ mas or less). Therefore, the best method to reveal their properties is frequent high-resolution and high signal-to-noise ratio spectroscopy aided by multicolor photometry and spectropolarimetry. In addition, near- and mid-IR imaging and interferometry may reveal the structure of the objects' dusty envelopes.

FS CMa stars along with their higher luminosity $\mathrm{sgB}[\mathrm{e}]$ counterparts seem to be important in many respects. In general, hot stars are considered to be significant contributors of gas to the IS medium (comparable to supernovae; Castor 1993). Similarly, they could supply a large amount of dust. According to studies of dust formation in the Milky Way, WR stars may account for $\sim 1 \%$ of the dust produced in CS environments (Gehrz 1989; Dwek 1998). These studies did not even consider B-type stars as dust producers. The wide luminosity range of the Galactic dust-forming B[e] stars $\left[2.5 \leq \log \left(L / L_{\odot}\right) \leq 5.1\right]$ and their location on the HRD mostly within the main sequence suggest that dust formation near hot stars is much more common than previously thought (Fig. 2).

The luminosity range of FS CMa stars alone $[2.5 \leq$ $\left.\log \left(L / L_{\odot}\right) \leq 4.5\right]$ indicates a wide range of their initial masses and suggests that they should largely outnumber more massive WR stars, only 24 of which ( $\sim 10 \%$ of all Galactic WR stars; van der Hucht 2001) are known to produce dust. Also, the dust formation rate in $\mathrm{B}[\mathrm{e}]$ stars with only a moderate IR excess ( $\mathrm{RY}$ Sct;
Men'shchikov \& Miroshnichenko 2005) seems to be an order of magnitude higher than in WR stars (Zubko 1998). Therefore, FS $\mathrm{CMa}$ and $\mathrm{sgB}[\mathrm{e}]$ stars may be responsible for at least a few percent of the Galactic CS dust.

Furthermore, comparison of the HRD locations of FS CMa stars with both binary (Wellstein et al. 2001) and single star models (Schaller et al. 1992) shows that it takes them $(5-30) \times 10^{6} \mathrm{yr}$ from the beginning of the main-sequence stage to reach the dust-forming stage. Since conditions near them are less "harsh" (less UV radiation due to lower $T_{\text {eff }}$ ) than those near WR stars, where dust can survive for $\sim 100 \mathrm{yr}$ (Marchenko et al. 2002), and the dust-forming $\mathrm{B}[\mathrm{e}]$ stars exist in the lower metallicity environments of the LMC and SMC (Zickgraf et al. 1986), it is reasonable to suggest that they might have been important dust producers in the earlier universe.

In this paper analysis of the properties of the most prominent (the brightest and those with the strongest IR excesses) dust-forming $\mathrm{B}[\mathrm{e}]$ stars is presented. It served as a basis for a new study of the IRAS PSC in an attempt to find more such objects. Nearly 30 new B[e] star candidates were found by Miroshnichenko et al. (2003c). Our recent observations, which will be reported in the next paper of this series, have proved that most of them are indeed dust-forming B[e] stars (mostly FS CMa stars).

I acknowledge support from NASA grant NAG5-8054. This research has made use of the SIMBAD database operated at CDS, Strasbourg, France.
Allen, D. A., \& Swings, J.-P. 1976, A\&A, 47, 293

Aumann, H. H. 1985, PASP, 97, 885

Baines, D., Oudmaijer, R. D., Porter, J. M., \& Pozzo, M. 2006, MNRAS, 367, 737

Barsukova, E. A., Borisov, N. V., Burenkov, A. N., Klochkova, V. G., Goranskij, V. P., \& Metlova, N. V. 2006, Astron. Rep., 50, 664

Bjorkman, J. E. 1998, in B[e] Stars, ed. A.-M. Hubert \& C. Jaschek (Dordrecht: Kluwer), 189

Bjorkman, K. S., Miroshnichenko, A. S., McDavid, D. A., \& Pogrosheva, T. M. 2002, ApJ, 573, 812

Blöcker, T. 1995, A\&A, 299, 755

Carlson, E., \& Henize, K. G. 1979, Vistas Astron., 23, 213

Castor, J. I. 1993, in ASP Conf. Ser. 35, Massive Stars: Their Lives in the Interstellar Medium, ed. J. P. Cassinelli \& J. B. Churchwell (San Francisco: ASP), 297

Castro-Carrizo, A., Bujarrabal, V., Sánchez Contreras, C., Alcolea, J., \& Neri, R. 2002, A\&A, 386, 633

Cidale, L., Zorec, J., \& Tringaniello, L. 2001, A\&A, 368, 160

Clark, J. S., et al. 2000, A\&A, 356, 50

Cutri, R. M., et al. 2003, CDS/ADC Collection of Electronic Catalogues, 2246 de Freitas Pacheco, J. A., Gilra, D. P., \& Pottasch, S. R. 1982, A\&A, 108, 111 de Jager, C., Nieuwenhuijzen, H., \& van der Hucht, K. A. 1988, A\&AS, 72, 259

Dong, Y. S., \& Hu, J. Y. 1991, Chinese Astron. Astrophys., 15, 275

Dwek, E. 1998, ApJ, 501, 643

Egan, M. P., et al. 2003, The Midcourse Space Experiment Point Source Catalog, Version 2.3, AFRL-VS-TR-2003

Finkenzeller, U., \& Mundt, R. 1984, A\&AS, 55, 109

Gehrz, R. D. 1989, in IAU Symp. 135, Interstellar Dust, ed. L. J. Allamandola \& A. G. G. M. Tielens (Dordrecht: Kluwer), 445

Geisel, S. L. 1970, ApJ, 161, L105

Grady, C. A., et al. 1996, A\&AS, 120, 157

Grinin, V. P., Kiselev, N. N., Chernova, G. P., Minikulov, N. Kh., \& Voshchinnikov, N. V. 1991, Ap\&SS, 186, 283

Herbig, G. H. 1994, in ASP Conf. Ser. 62, The Nature and Evolutionary Status of Herbig Ae/Be Stars, ed. P. S. Thé, M. R. Peréz, \& E. P. J. van den Heuvel (San Francisco: ASP), 3

Hillier, D. J. 2006, in ASP Conf. Ser. 355, Stars with the B[e] Phenomenon, ed. M. Kraus \& A. S. Miroshnichenko (San Francisco: ASP), 39

Hofmann, K.-H., Balega, Y., Ikhsanov, N. R., Miroshnichenko, A. S., \& Weigelt, G. 2002, A\&A, 395, 891

\section{EFERENCES}

Hollenbach, D., \& Adams, F. C. 2004, in ASP Conf. Ser. 324, Debris Disks and the Formation of Planets: A Symposium in Memory of Fred Gillett, ed. L. Caroff et al. (San Francisco: ASP), 168

IPAC 1986, IRAS Catalog of Point Sources, Version 2.0, Joint IRAS Science

Ivezić, Ž., Miroshnichenko, A. S., \& Elitzur, M. 1998, in B[e] Stars, ed. A.-M. Hubert \& C. Jaschek (Dordrecht: Kluwer), 227

Kraus, M., \& Lamers, H. J. G. L. M. 2003, A\&A, 405, 165

Křiž, S., \& Harmanec, P. 1975, Bull. Astron. Inst. Czechoslovakia, 26, 65

Lamers, H. J. G. L. M., Zickgraf, F.-J., de Winter, D., Houziaux, L., \& Zorec, J. 1998, A\&A, 340, 117 (L98)

Landaberry, S. J. C., Pereira, C. B., \& de Araújo, F. X. 2001, A\&A, 376, 917

Low, F. J., Johnson, H. L., Kleinmann, D. E., Latham, A. S., \& Geisel, S. L. 1970, ApJ, 160, 531

Malfait, K., Bogaert, E., \& Waelkens, C. 1998, A\&A, 331, 211

Marchenko, S. V., Moffat, A. F. J., Vacca, W. D., Côté, S., \& Doyon, R. 2002, ApJ, 565, L59

Men'shchikov, A. B., \& Miroshnichenko, A. S. 2005, A\&A, 443, 211

Meyer, J. M., Nordsieck, K. H., \& Hoffman, J. L. 2002, AJ, 123, 1639

Meynet, G., \& Maeder, A. 2006, in ASP Conf. Ser. 355, Stars with the B[e] Phenomenon, ed. M. Kraus \& A. S. Miroshnichenko (San Francisco: ASP), 27

Miroshnichenko, A. S. 2006, in ASP Conf. Ser. 355, Stars with the B[e] Phenomenon, ed. M. Kraus \& A. S. Miroshnichenko (San Francisco: ASP), 13

Miroshnichenko, A. S., Bergner, Yu. K., Kuratov, K. S., Mukanov, D. B., \& Sheikina, T. A. 1996, Astron. Rep., 40, 509

Miroshnichenko, A. S., Bjorkman, K. S., Chentsov, E. L., \& Klochkova, V. G. 2002a, in ASP Conf. Ser. 279, Exotic Stars as Challenges to Stellar Evolution, ed. C. A. Tout \& W. Van Hamme (San Francisco: ASP), 303

Miroshnichenko, A. S., Bjorkman, K. S., Grosso, M., Hinkle, K., \& Levato, H. 2005, A\&A, 436, 653

Miroshnichenko, A. S., \& Corporon, P. 1999, A\&A, 349, 126

Miroshnichenko, A. S., Ivezić, Ž., Vinković, D., \& Elitzur, M. 1999, ApJ, 520, L115

Miroshnichenko, A. S., Klochkova, V. G., \& Bjorkman, K. S. 2003a, Astron. Lett., 29, 336

Miroshnichenko, A. S., Klochkova, V. G., Bjorkman, K. S., \& Panchuk, V. E. 2002b, A\&A, 390, 627

Miroshnichenko, A. S., Levato, H., Bjorkman, K. S., \& Grosso, M. 2001, A\&A, 371, 600 2003b, A\&A, 406, 673 
Miroshnichenko, A. S., et al. 2000, A\&AS, 147, 5

2002c, A\&A, 383, 171

2002d, A\&A, 388, 563

2003c, BAAS, 35, 1359

2004, A\&A, 417, 731

2006, in ASP Conf. Ser. 355, Stars with the B[e] Phenomenon, ed. M.

Kraus \& A. S. Miroshnichenko (San Francisco: ASP), 347

Monet, D. G., et al. 2003, AJ, 125, 984

Pereira, C. B., Franco, C. S., \& de Araújo, F. X. 2003, A\&A, 397, 927

Podsiadlowski, Ph., Morris, T. S., \& Ivanova, N. 2006, in ASP Conf. Ser. 355, Stars with the B[e] Phenomenon, ed. M. Kraus \& A. S. Miroshnichenko (San Francisco: ASP), 259

Pogodin, M. A., Franco, G. A. P., \& Lopes, D. F. 2005, A\&A, 438, 239

Pogodin, M. A., et al. 2004, A\&A, 417, 715

Prinja, R. K. 1989, MNRAS, 241, 721

Robinson, E. L., Ivans, I. I., \& Welsh, W. F. 2002, ApJ, 565, 1169

Sabbadin, F., \& Bianchini, A. 1979, PASP, 91, 278

Salpeter, E. E. 1974, ApJ, 193, 585

Schaller, G., Schaerer, D., Meynet, G., \& Maeder, A. 1992, A\&AS, 96, 269

Sheikina, T. A., Miroshnichenko, A. S., \& Corporon, P. 2000, in ASP Conf. Ser. 214, The Be Phenomenon in Early-Type Stars, ed. M. A. Smith, H. F. Henrichs, \& J. Fabregat (San Francisco: ASP), 494

Sitko, M. L., Halbedel, E. M., Lawrence, G. F., Smith, J. A., \& Janow, K. 1994, ApJ, 432, 753

Stee, Ph., Bittar, J., \& Lopez, B. 2004, ApJ, 602, 978
Stenholm, B., \& Acker, A. 1987, A\&AS, 68, 51

Suárez, O., García-Lario, P., Manchado, A., Manteiga, M., Ulla, A., \& Pottasch, S. R. 2006, A\&A, 458, 173

Swings, J.-P. 2006, in ASP Conf. Ser. 355, Stars with the B[e] Phenomenon, ed.

M. Kraus \& A. S. Miroshnichenko (San Francisco: ASP), 3

van der Hucht, K. A. 2001, NewA Rev., 45, 135

van der Hucht, K. A., Williams, P. M., \& Thé, P. S. 1984, in IAU Symp. 105,

Observational Tests of the Stellar Evolution Theory, ed. A. Maeder \& A.

Renzini (Dordrecht: Reidel), 273

van der Veen, W. E. C. J., Habing, H. J., \& Geballe, T. R. 1989, A\&A, 226, 108

Vink, J., de Koter, A., \& Lamers, H. J. G. L. M. 2001, A\&A, 369, 574

Wackerling, L. R. 1970, MmRAS, 73, 153

Wellstein, S., Langer, N., \& Braun, H. 2001, A\&A, 369, 939

Zamanov, R. K., Bode, M. F., Melo, C. H. F., Porter, J. M., Gomboc, A., \&

Konstantinova-Antova, R. 2006, MNRAS, 365, 1215

Zickgraf, F.-J. 2001, A\&A, 375, 122 2003, A\&A, 408, 257

2006, in ASP Conf. Ser. 355, Stars with the B[e] Phenomenon, ed. M.

Kraus \& A. S. Miroshnichenko (San Francisco: ASP), 135

Zickgraf, F.-J., Wolf, B., Stahl, O., Leitherer, C., \& Appenzeller, I. 1986, A\&A, 163,119

Zickgraf, F.-J., Wolf, B., Stahl, O., Leitherer, C., \& Klare, G. 1985, A\&A, 143, 421

Zubko, V. G. 1998, MNRAS, 295, 109 


\section{AUTHOR QUERIES}

Q1 Au: Your article has been edited for grammar, consistency, and to conform to journal style (see Instructions to Authors). To expedite publication, we generally do not query every routine grammatical and style change made to a manuscript, although all substantive changes have been noted. Please review the article carefully. Note that we may be unable to make changes that conflict with journal style, obscure meaning, or create grammatical or other problems. Also note that article proofs via PDF do not show corrections that will have been made by the typesetter's proofreader, which generally include corrections of errors of page layout, figure placement, and spacing and font mistakes; feel free to mark any errors you notice in these areas. When sending us your corrections, if you are writing them by hand, please print clearly. If sending a fax, please do not write too close to the margins of the page, as these are often cut off in fax transmission. Finally, please note that a delayed, incomplete, or illegible response may delay the publication of your article while we contact you. 